\title{
Inventory Time Reduction in Gen2 with Single-Antenna Separation of FM0 RFID Signals
}

\author{
John Kimionis*, Aggelos Bletsas*, Antonis G. Dimitriou ${ }^{\dagger}$, and George N. Karystinos* \\ *Department of Electronic and Computer Engineering \\ Technical University of Crete, Greece, 73100 \\ Email: ikimionis@isc.tuc.gr, aggelos@telecom.tuc.gr, karystinos@telecom.tuc.gr \\ ${ }^{\dagger}$ Department of Electrical and Computer Engineering \\ Aristotle University of Thessaloniki, Greece, 54124 \\ Email: antodimi@auth.gr
}

\begin{abstract}
In this work single-antenna detection schemes for collided radio frequency identification (RFID) signals are proposed to achieve waveform separation and extract information when two RFID tags simultaneously transmit. Industry standard Generation 2 (Gen2) tags with FM0 (biphase-space) encoding are fully exploited, including FMO inherent memory, in sharp contrast to tag separation prior art. It is shown that memory-assisted detection for two collided FMO tags performs $3 \mathrm{~dB}$ better in terms of bit-error-rate (BER), compared to memoryless detection. Information from waveform separation is used to significantly reduce the total inventory time for a given tag population in framed Aloha schemes, including the widely adopted Gen2. No modification of the existing readers' RF front end is required, as the proposed techniques could operate with single-antenna readers. This might be important for portable UHF Gen2 RFID systems, where multiple-antennas are not always an option.
\end{abstract}

\section{INTRODUCTION}

A large amount of research work and commercial applications on the field of radio frequency identification (RFID) technology has been presented the last years. Backscattering of signals (i.e. signal reflection and not active radiation [1]), is an appealing scheme for information transmission, where both low-cost and energy efficiency matter. Nowadays, large amounts of RFID tags are used in supply chains for product identification [2], or sensor networks [3]-[5], where the two aforementioned conditions (cost and energy) are critical. One of the biggest RFID challenges is the capability to resolve collisions, i.e. simultaneous transmission of information from more than one tags.

The current industry standard EPC Class 1 Generation 2 (or ISO 18000-6C) [6], specifies collision resolution through framed Aloha schemes, where each tag randomly claims one slot of the frame for data transmission [7], [8]. Collision occurs when two or more tags claim the same slot, and so transmit data simultaneously, resulting in a waveform which is nondetectable with standard (i.e. single-tag) detection techniques at the RFID reader. Furthermore, no action is taken for these particular tags until the next frame (inventory round) begins. Although Gen2 specifies the framed Aloha scheme, no specification exists on the detection that takes place on the RFID reader, thus leaving the subject of separating collided tag information open.
Recent work has been presented on the subject of RFID waveform separation, with one of the first being [9], which utilized a software-defined monitor for non-Gen2 RFID tags, and tested separation on DBPSK modulation. Processing of the in-phase (I) and quadrature (Q) signals in high signal-to-noise ratio (SNR), when non-Gen2 tags simultaneously transmitted was presented in [10]. Modeling of the backscatter radio channel and the received I and Q components were exploited in [11] with zero-forcing techniques. Throughput enhancement of framed Aloha was also calculated. Finally, multi-antenna detection, based on blind source separation of zero constantmodulus signals was proposed in [12] and experimentally validated in [13].

However, prior art did not take into account the FM0 tag encoding scheme (one of the two encoding schemes used in Gen2 standard) and its inherent memory. Furthermore, some of the proposed techniques required multiple antennas, which may not be always a valid option (e.g. in portable UHF systems). In this work, we fully exploit the encoding characteristics of FM0 and derive BER-optimal detectors using singleantenna techniques for collided tag information. Continuing the work in [14], we follow the same signal model, extend the detection technique for two tags and validate theoretical results by simulation.

Total delay (and not throughput) of the framed Aloha scheme is calculated when single-tag detection is performed (i.e. conventional reader processing) and is compared with the new, reduced delay when two-tag separation can be utilized. Inventory delay of a given tag population is measured in slots, which is more important than throughput, as the length of data transmitted by RFID tags is fixed. The methods proposed can be readily applied in current RFID Gen2 readers, without modification of their RF front ends, since they do not rely on multiple antennas for tag separation.

Section II formulates the problem and describes the system model that is followed in this work. Section III describes the techniques proposed for both memoryless and memoryassisted detection. In Section IV, total inventory delay for a given tag population is calculated, when the proposed detection techniques are applied. Numerical results are presented in Section V and finally, Section VI offers the conclusion of this 


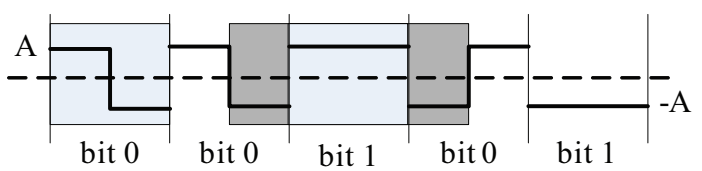

Fig. 1. Baseband FM0 signal of a single tag. Levels always change at the bit interval. For bit " 0 ," level also changes at the middle of the bit period.

work.

\section{Problem Formulation and System Model}

In FM0 encoding, signal (line) level always changes at the bit boundaries. Moreover, signal level changes at the middle of the bit period only for bit " 0 " (while for bit " 1 " the level is kept constant) as depicted in Fig. 1. Thus, encoding of a single FM0 bit requires memory of the previous bit so that signal levels are modified accordingly at the bit boundaries. Each FM0-encoded bit can be represented as a vector of two half-bit constants of the form $\left[\begin{array}{ll} \pm a & \pm a\end{array}\right]^{T}$ where sign of $a$ depends on the transmitted bit as well as the signal memory (i.e. previous transmission level).

To validate the signal model of [14] that we follow in this work, we utilized a simple and low-cost measurement setup (Fig. 3-(a)) that consists of a commercial UHF Gen2 reader, two FM0 tags, and a USRP software-defined radio (SDR) with a broadband daughterboard tuned at $865 \mathrm{MHz}$; the SDR acts as a low-cost Gen2 monitor (sniffer). A SDR-based Gen2 monitor was also recently developed in [15]. With custom software developed throughout this work, conversation between two tags and the reader was recorded at the sniffer. The downconverted baseband signal magnitude $\sqrt{I^{2}(t)+Q^{2}(t)}$ at the sniffer (where $I(t)$ and $Q(t)$ represent the in-phase and quadrature, respectively, signal components) is depicted in Fig. 3-(b) where it is shown that on top of a DC constant there is encoded information (due to the carrier transmitted from the reader and scattered back from the tags). The signal part depicted as "collision" is magnified and zero-centered in Fig. 3-(c) which depicts the measured downconverted sum of two FM0 signals; such "collision" corresponds to simultaneous transmission (through backscatter) during the query phase of the Gen2 protocol, when random 16-bit ID information is transmitted by each tag (a.k.a. RN16). The above measurement validates the signal model of [14] followed in this work; furthermore, processing of $\sqrt{I^{2}(t)+Q^{2}(t)}$ eliminates the frequency offset between reader and sniffer. It is noted however that at an operating RFID reader, where the detection methods proposed in this work could be implemented, there is no frequency offset between the reader's transmit and receive paths (i.e. the reader uses the same oscillator for up- and downconversion) [16].

Consequently, after pulse-matched filtering and sampling at the RFID reader, the in-phase (or quadrature) ${ }^{1}$ component of

\footnotetext{
${ }^{1}$ For simplicity of the derivations and clarity of the presentation, in this work we consider processing of the in-phase (or quadrature) component only. Our developments can be extended to joint processing of the in-phase and quadrature components in a straightforward manner.
}

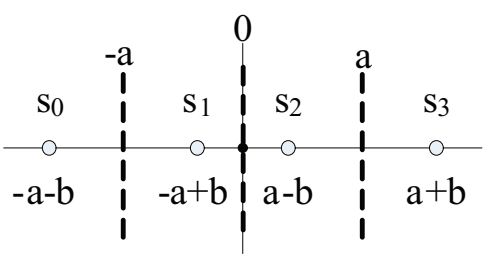

Fig. 2. The two-tag, nonuniform signal constellation with decision areas (marked with intermittent lines) based on the minimum distance rule.

the collided signal during one bit period can be represented by a vector $\left[x_{0} x_{1}\right]^{T}$ of two half-bit symbols, where each half-bit symbol belongs in $\mathcal{S}=\left\{s_{0}=-a-b, s_{1}=-a+b, s_{2}=\right.$ $\left.a-b, s_{3}=a+b\right\}$. Slow-fading can be assumed, i.e. $a, b$ remain constant during reception given the limited number of considered bits, either in RN16 or in the actual tag ID ( 96 bits in electronic product code). We also assume coherent reception, i.e. the constants $a, b$ are considered known at the receiver. Such knowledge can be acquired through estimation using specialized pilot signals. It is remarked that, if $a=b$, then $s_{1}=s_{2} \in \mathcal{S}$ and information is lost, i.e. separation of tags $\mathrm{A}$ and $\mathrm{B}$ fails. In general, $a \neq b$ and their difference will be explicitly taken into account. Without loss of generality, we assume $a>b$ throughout this work.

Under the above assumptions, the received signal can be written in vector form as:

$$
\mathbf{y} \triangleq\left[\begin{array}{l}
y_{0} \\
y_{1}
\end{array}\right]=\left[\begin{array}{l}
x_{0} \\
x_{1}
\end{array}\right]+\mathbf{n},
$$

where $\left[\begin{array}{ll}x_{0} & x_{1}\end{array}\right]^{T} \in \mathcal{S}^{2}$ is the collided information signal and $\mathbf{n}=\left[\begin{array}{ll}n_{0} & n_{1}\end{array}\right]^{T}$ represents additive white Gaussian noise (AWGN) where $n_{0}, n_{1}$ are independent, zero-mean Gaussian variables with variance $\sigma^{2}$.

The minimum distance rule (ML) given measurement $y_{i}, i \in$ $\{0,1\}$ and transmitted constellation $\mathcal{S}$, with decision boundaries depicted in Fig. 2, provides the following conditional error probability:

$$
\begin{aligned}
\operatorname{Pr}\left(\hat{x}_{i} \neq x_{i} \mid x_{i}=s_{0}\right) & =\operatorname{Pr}\left(\hat{x}_{i} \neq x_{i} \mid x_{i}=s_{3}\right) \\
& =Q(b / \sigma), i=0,1 \\
\operatorname{Pr}\left(\hat{x}_{i} \neq x_{i} \mid x_{i}=s_{1}\right) & =\operatorname{Pr}\left(\hat{x}_{i} \neq x_{i} \mid x_{i}=s_{2}\right) \\
& =Q((a-b) / \sigma)+Q(b / \sigma), i=0,1,
\end{aligned}
$$

where $Q(x)=\frac{1}{\sqrt{2 \pi}} \int_{x}^{+\infty} e^{-t^{2} / 2} d t$ is the $\mathrm{Q}$ function. The expressions above will be found useful throughout the document.

The above modeling approach is sufficient for the examination of the proposed two-tag detection methods. For complex modeling of the backscatter radio channel, the interested reader could refer to several works, including [3], [11], and [17].

\section{Detection Techniques}

In subsections III-A and III-B, we derive different methods for detection of both tag A and tag B FM0 information or only tag A information (in the presence of B), alongside with their respective bit-error-rate (BER) probabilities. 


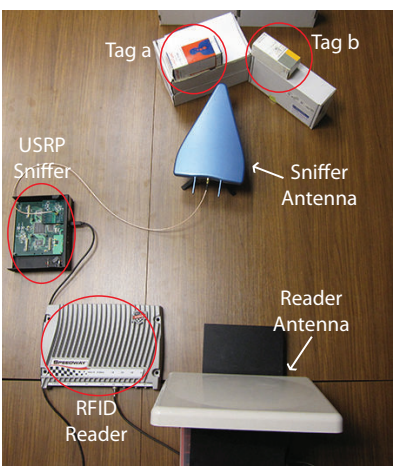

(a) The custom measurement setup.

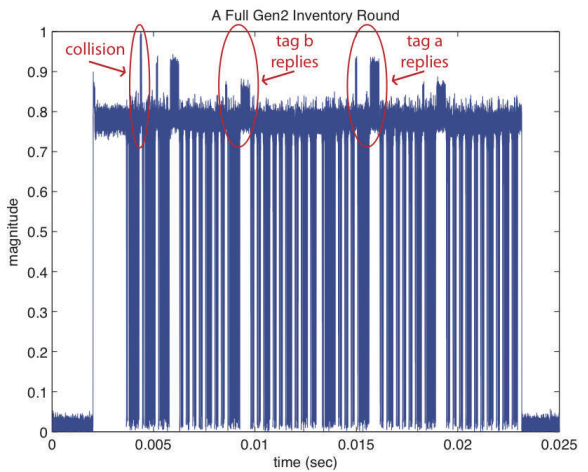

(b) Conversation between reader and two tags captured at the SDR sniffer.

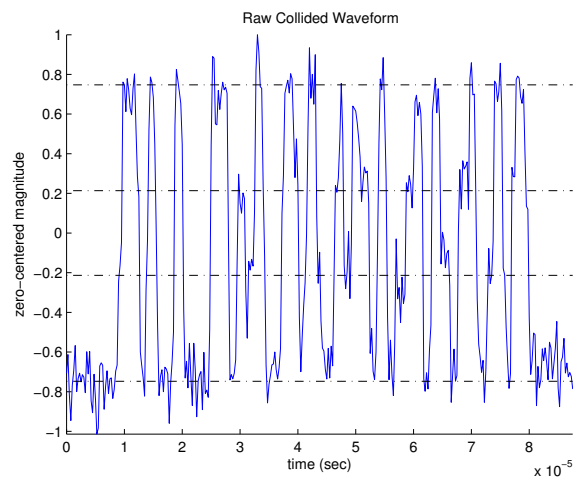

(c) Zero-centered magnitude of the received waveform for two collided FM0 tags.

Fig. 3. Baseband received signal at the sniffer with conversation between reader and two tags. The signal depicted as "collision" in the second figure is magnified and zero-centered in the third figure and depicts two collided FM0 signals from two tags.

\section{A. Memoryless Detection of Collided Tags Information}

This method performs independent detection of the two half-bit symbols (according to decision areas of Fig. 2) and then, based on the findings, final decision on both tag $\mathrm{A}$ and $\mathrm{B}$ information is jointly made. The detection method is summarized below:

- Detect $\hat{x}_{0} \in \mathcal{S}$ from $y_{0}$, applying a ML (i.e. minimumdistance) rule.

- Detect $\hat{x}_{1} \in \mathcal{S}$ from $y_{1}$, applying a ML (i.e. minimumdistance) rule.

- Decide in favor of $\mathrm{H}_{i}$ (i.e. $\hat{\mathrm{H}}=\mathrm{H}_{i}$ ), $i \in\{0,1,2,3\}$, from sign change between $\hat{x}_{0}$ and $\hat{x}_{1}$. If sign of $a$ in $\hat{x}_{0}$ is different than in $\hat{x}_{1}$, then $\widehat{\operatorname{tag}}_{\mathrm{A}}=0$, otherwise $\widehat{\operatorname{tag}}_{\mathrm{A}}=1$. Similarly, if sign of $b$ in $\hat{x}_{0}$ is different than in $\hat{x}_{1}$, then $\widehat{\operatorname{tag}}_{\mathrm{B}}=0$, otherwise $\widehat{\operatorname{tag}}_{\mathrm{B}}=1$.

For example, if $\hat{x}_{0}=a-b=s_{2}$ and $\hat{x}_{1}=-a-b=s_{0}$, then the bit estimates for tags A and B are $\operatorname{tag}_{\mathrm{A}}=0$ and $\operatorname{tag}_{\mathrm{B}}=1$, respectively. Such a case corresponds to hypothesis $\mathrm{H}_{2}$ according to Table I-A. It is remarked that Method 1 does not require knowledge of the noise variance $\sigma^{2}$ per half-bit, at the receiver.

It is straightforward to compute error (or, equivalently, zeroerror) performance of the above detection method. Observing that, under hypothesis $\mathrm{H}_{0}$ and FM0 signaling, only transitions between $s_{0}$ and $s_{3}$ or between $s_{1}$ and $s_{2}$ are allowed, the following conditional error probability can be readily calculated.

$$
\begin{aligned}
& \operatorname{Pr}\left(\hat{\mathrm{H}}_{i}, i \neq 0 \mid \mathrm{H}_{0}\right)=1-\operatorname{Pr}\left(\hat{\mathrm{H}}_{0} \mid \mathrm{H}_{0}\right)= \\
& 1-\frac{1}{4}\left\{\left[1-\operatorname{Pr}\left(\hat{x}_{0} \neq x_{0} \mid x_{0}=s_{0}\right)\right]\left[1-\operatorname{Pr}\left(\hat{x}_{1} \neq x_{1} \mid x_{1}=s_{3}\right)\right]\right. \\
& \quad+\left[1-\operatorname{Pr}\left(\hat{x}_{0} \neq x_{0} \mid x_{0}=s_{1}\right)\right]\left[1-\operatorname{Pr}\left(\hat{x}_{1} \neq x_{1} \mid x_{1}=s_{2}\right)\right] \\
& \quad+\left[1-\operatorname{Pr}\left(\hat{x}_{0} \neq x_{0} \mid x_{0}=s_{2}\right)\right]\left[1-\operatorname{Pr}\left(\hat{x}_{1} \neq x_{1} \mid x_{1}=s_{1}\right)\right] \\
& \left.\quad+\left[1-\operatorname{Pr}\left(\hat{x}_{0} \neq x_{0} \mid x_{0}=s_{3}\right)\right]\left[1-\operatorname{Pr}\left(\hat{x}_{1} \neq x_{1} \mid x_{1}=s_{0}\right)\right]\right\} \\
& =1-\frac{1}{2}\left\{[1-Q(b / \sigma)]^{2}+[1-Q(b / \sigma)-Q((a-b) / \sigma)]^{2}\right\} .
\end{aligned}
$$

Under hypothesis $\mathrm{H}_{1}$ and FM0 signaling, transitions between $s_{0}$ and $s_{1}$ or between $s_{2}$ and $s_{3}$ are allowed. Thus,

$$
\begin{aligned}
& \operatorname{Pr}\left(\hat{\mathrm{H}}_{i}, i \neq 1 \mid \mathrm{H}_{1}\right)=1-\operatorname{Pr}\left(\hat{\mathrm{H}}_{1} \mid \mathrm{H}_{1}\right)= \\
& 1-\frac{1}{4}\left\{\left[1-\operatorname{Pr}\left(\hat{x}_{0} \neq x_{0} \mid x_{0}=s_{0}\right)\right]\left[1-\operatorname{Pr}\left(\hat{x}_{1} \neq x_{1} \mid x_{1}=s_{1}\right)\right]\right. \\
& \quad+\left[1-\operatorname{Pr}\left(\hat{x}_{0} \neq x_{0} \mid x_{0}=s_{1}\right)\right]\left[1-\operatorname{Pr}\left(\hat{x}_{1} \neq x_{1} \mid x_{1}=s_{0}\right)\right] \\
& \quad+\left[1-\operatorname{Pr}\left(\hat{x}_{0} \neq x_{0} \mid x_{0}=s_{2}\right)\right]\left[1-\operatorname{Pr}\left(\hat{x}_{1} \neq x_{1} \mid x_{1}=s_{3}\right)\right] \\
& \left.\quad+\left[1-\operatorname{Pr}\left(\hat{x}_{0} \neq x_{0} \mid x_{0}=s_{3}\right)\right]\left[1-\operatorname{Pr}\left(\hat{x}_{1} \neq x_{1} \mid x_{1}=s_{2}\right)\right]\right\} \\
& =1-\{[1-Q(b / \sigma)][1-Q(b / \sigma)-Q((a-b) / \sigma)]\}
\end{aligned}
$$

Under similar reasoning, it can be shown that:

$$
\begin{aligned}
& \operatorname{Pr}\left(\hat{\mathrm{H}}_{i}, i \neq 2 \mid \mathrm{H}_{2}\right)=\operatorname{Pr}\left(\hat{\mathrm{H}}_{i}, i \neq 1 \mid \mathrm{H}_{1}\right), \\
& \operatorname{Pr}\left(\hat{\mathrm{H}}_{i}, i \neq 3 \mid \mathrm{H}_{3}\right)=\operatorname{Pr}\left(\hat{\mathrm{H}}_{i}, i \neq 0 \mid \mathrm{H}_{0}\right) .
\end{aligned}
$$

Therefore, probability of detection error in at least one of the two tags is given by:

$$
\begin{gathered}
\operatorname{Pr}\left(\left(\operatorname{tag}_{\mathrm{A}}, \operatorname{tag}_{\mathrm{B}}\right) \neq\left(\operatorname{tag}_{\mathrm{A}}, \operatorname{tag}_{\mathrm{B}}\right)\right)=\frac{1}{4} \sum_{j=0}^{3} \operatorname{Pr}\left(\hat{\mathrm{H}}_{i}, i \neq j \mid \mathrm{H}_{j}\right) \\
=Q\left(\frac{b}{\sigma}\right)\left[2-Q\left(\frac{b}{\sigma}\right)-Q\left(\frac{a-b}{\sigma}\right)\right] \\
+Q\left(\frac{a-b}{\sigma}\right)\left[1-\frac{1}{4} Q\left(\frac{a-b}{\sigma}\right)\right]
\end{gathered}
$$

If we restrict the definition of detection error solely with respect to tag A, i.e. correct (or erroneous) detection of tag $\mathrm{B}$ is indifferent, and follow the above method, then the error probability can be also readily calculated. Decision areas for half-bit detection in Fig. 2 become $\left(y_{i}<0\right.$ for $\hat{x}_{i}=s_{0}$ or $s_{1}$ and $y_{i}>0$ for $\hat{x}_{i}=s_{2}$ or $\left.s_{3}, i \in\{0,1\}\right)$ and conditional 
error probabilities of eqs. (2) and (3) are modified to:

$$
\begin{aligned}
\operatorname{Pr}\left(\hat{x}_{i}=s_{2} \text { or } s_{3} \mid x_{i}=s_{0}\right) & =\operatorname{Pr}\left(\hat{x}_{i}=s_{0} \text { or } s_{1} \mid x_{i}=s_{3}\right) \\
& =Q\left(\frac{a+b}{\sigma}\right), \quad i=0,1 \\
\operatorname{Pr}\left(\hat{x}_{i}=s_{2} \text { or } s_{3} \mid x_{i}=s_{1}\right) & =\operatorname{Pr}\left(\hat{x}_{i}=s_{0} \text { or } s_{1} \mid x_{i}=s_{2}\right) \\
& =Q\left(\frac{a-b}{\sigma}\right), \quad i=0,1 .
\end{aligned}
$$

Following the same derivation of eqs. (4)-(7), the bit error probability of detection of tag A information with the above (memoryless) method becomes:

$$
\begin{aligned}
\operatorname{Pr}\left(\widehat{\operatorname{tag}}_{\mathrm{A}}\right. & \left.\neq \operatorname{tag}_{\mathrm{A}}\right)=\left[Q\left(\frac{a+b}{\sigma}\right)+Q\left(\frac{a-b}{\sigma}\right)\right] \\
& \times\left\{1-\frac{1}{4}\left[Q\left(\frac{a+b}{\sigma}\right)+Q\left(\frac{a-b}{\sigma}\right)\right]\right\} .
\end{aligned}
$$

B. One-Bit-Memory-Assisted Detection of Collided Tags Information

The previous method focused on the duration of a single bit (two consecutive half-bits) and, therefore, did not exploit the inherent memory of FM0 signaling. in this method, memory of FM0 signaling is exploited in detection of two collided FM0 signals by observing duration of exactly two bits: the bit under observation, half-bit before it, and half-bit after. Similar mindset was exploited by Simon and Divsalar [14] for detection of a single tag. They noticed that for ML single-bit (memoryless) detection there are four possible hypotheses to test; however, if half-bit before and half bit after are also observed, then there are only two hypotheses at the bit boundary (see shaded half-bits at Fig. 1). Below, we extend the idea in detection and separation of two FM0 tags.

With slight abuse of notation, we denote by $y_{0}$ the received half-bit signal before the bit boundary and $y_{1}$ the received half-bit signal after the bit boundary. Thus, there is a pair of measurements $\left(y_{0}, y_{1}\right)^{0}$ where $y_{1}$ corresponds to the first halfbit and $y_{0}$ corresponds to the second half-bit of the previous bit and a second pair of measurements $\left(y_{0}, y_{1}\right)^{1}$ where $y_{0}$ corresponds to the second half-bit and $y_{1}$ corresponds to the first half-bit of the next bit.

Given that the FM0 signal of each tag always changes levels at the bit boundaries, the possible transmitted symbols $s_{0}, s_{1}, s_{2}$, and $s_{3}$ under either pair of measurements $\left(y_{0}, y_{1}\right)^{i}, i=0,1$, are depicted in Figures 5-a and 5-b. The detection algorithm works as follows.

- Detect $\hat{x}_{0} \in \mathcal{S}$ from $\left(y_{0}, y_{1}\right)^{0}$, applying a ML (i.e. minimum-distance) rule (Fig. 5-a).

- Detect $\hat{x}_{1} \in \mathcal{S}$ from $\left(y_{0}, y_{1}\right)^{1}$, applying a ML (i.e. minimum-distance) rule (Fig. 5-b).

- Decide in favor of $\mathrm{H}_{i}, i=0,1,2,3$, based on $\hat{x}_{0}, \hat{x}_{1}$, according to Table I-B.

For example, if $\hat{x}_{0}=s_{2}$ and $\hat{x}_{1}=s_{0}$, then tag $\mathrm{B}$ level remains constant at $-b$ (i.e. bit " 1 ") while tag A level switches from $+a$ to $-a$ (i.e. bit " 0 "). Thus, we decide in favor of hypothesis $\mathrm{H}_{2}$, according to Table I-right. Similarly, the other entries above can be worked out.
The ML (i.e. minimum-distance) rule for $\left(y_{0}, y_{1}\right)^{0}$ or $\left(y_{0}, y_{1}\right)^{1}$ can be directly derived, by simple observation of the initial hypothesis, depicted at Fig. 5-a,Fig. 5-b. Working on $\left(y_{0}, y_{1}\right)^{0}$ and $\left(y_{0}, y_{1}\right)^{1}$, the distances for the four transmitted symbols $s_{0}, s_{1}, s_{2}, s_{3}$ are given by $d_{0}^{i}, d_{1}^{i}, d_{2}^{i}$, and $d_{3}^{i}, i=0,1$, respectively, that are equal to:

$d_{0}^{0}\left[y_{0}, y_{1}\right]=d_{3}^{1}\left[y_{0}, y_{1}\right]=\left[y_{0}-(a+b)\right]^{2}+\left[y_{1}-(-a-b)\right]^{2}$,

$d_{1}^{0}\left[y_{0}, y_{1}\right]=d_{2}^{1}\left[y_{0}, y_{1}\right]=\left[y_{0}-(a-b)\right]^{2}+\left[y_{1}-(-a+b)\right]^{2}$,

$d_{2}^{0}\left[y_{0}, y_{1}\right]=d_{1}^{1}\left[y_{0}, y_{1}\right]=\left[y_{0}-(-a+b)\right]^{2}+\left[y_{1}-(a-b)\right]^{2}$,

$d_{3}^{0}\left[y_{0}, y_{1}\right]=d_{0}^{1}\left[y_{0}, y_{1}\right]=\left[y_{0}-(-a-b)\right]^{2}+\left[y_{1}-(a+b)\right]^{2}$.

Using $\left(y_{0}, y_{1}\right)^{0}$ and the distances of $d_{0}^{0}, d_{1}^{0}, d_{2}^{0}$, and $d_{3}^{0}$, in the following we describe how decision on $\hat{x}_{0}$ is made. Similar approach is followed subsequently for the decision on $\hat{x}_{1}$ (based on $\left(y_{0}, y_{1}\right)^{1}$ and $d_{0}^{1}, d_{1}^{1}, d_{2}^{1}$, and $d_{3}^{1}$ ).

We detect $\hat{x}_{0}=s_{0}$ if and only if:

$$
\begin{aligned}
d_{0}^{0}<d_{1}^{0} & \Leftrightarrow y_{0}-y_{1}>2 a, \\
d_{0}^{0}<d_{2}^{0} & \Leftrightarrow y_{0}-y_{1}>2 b, \\
d_{0}^{0}<d_{3}^{0} & \Leftrightarrow y_{0}-y_{1}>0 .
\end{aligned}
$$

Having in mind that $a>b$, we obtain:

$$
\hat{x}_{0}=s_{0}: \quad y_{0}-y_{1}>2 a .
$$

Working similarly for the other three hypotheses, the ML decision areas become:

$$
\begin{aligned}
& \hat{x}_{0}=s_{1}: 0<y_{0}-y_{1}<2 a, \\
& \hat{x}_{0}=s_{2}:-2 a<y_{0}-y_{1}<0, \\
& \hat{x}_{0}=s_{3}: y_{0}-y_{1}<-2 a .
\end{aligned}
$$

The four decision areas are depicted in Fig. 4.

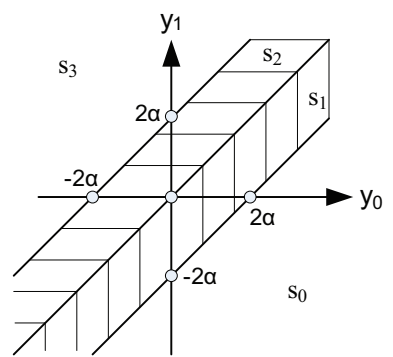

Fig. 4. Decision areas for each pair of measurements in memory-assisted detection.

Following similar steps, we can derive the corresponding decision rules for $\hat{x}_{1}$ (based on $\left(y_{0}, y_{1}\right)^{1}$ and $d_{0}^{1}, d_{1}^{1}, d_{2}^{2}$, and $d_{3}^{1}$ ) which are simplified to: 
TABLE I

\begin{tabular}{|c|c|c|c|c|c|c|c|c|c|c|c|c|c|c|c|c|c|c|c|c|c|}
\hline & $\mathrm{H}_{0}$ & $\mathrm{H}_{1}$ & $\mathrm{H}_{2}$ & $\mathrm{H}_{3}$ & $\hat{x}_{0}$ & $s_{0}$ & $s_{0}$ & $s_{0}$ & $s_{0}$ & $s_{1}$ & $s_{1}$ & $s_{1}$ & $s_{1}$ & $s_{2}$ & $s_{2}$ & $s_{2}$ & $s_{2}$ & $s_{3}$ & $s_{3}$ & $s_{3}$ & $s_{3}$ \\
\hline $\operatorname{tag}_{A}$ & $\overline{00}$ & $\overline{17}$ & $\overline{c 0}$ & $\bar{~}$ & $\hat{x}_{1}$ & $s_{0}$ & $s_{1}$ & $s_{2}$ & $s_{3}$ & $s_{0}$ & $s_{1}$ & $s_{2}$ & $s_{3}$ & $s_{0}$ & $s_{1}$ & $s_{2}$ & $s_{3}$ & $s_{0}$ & $s_{1}$ & $s_{2}$ & $s_{3}$ \\
\hline $\operatorname{tag}_{B}$ & 0 & 0 & 1 & 1 & $\hat{\mathrm{H}}$ & $\overline{\mathrm{H}_{3}}$ & $\overline{\mathrm{H}_{1}}$ & $\overline{\mathrm{H}_{2}}$ & $\overline{\mathrm{H}_{0}}$ & $\overline{\mathrm{H}_{1}}$ & $\overline{\mathrm{H}_{3}}$ & $\mathrm{H}_{0}$ & $\overline{\mathrm{H}_{2}}$ & $\mathrm{H}_{2}$ & $\overline{\mathrm{H}_{0}}$ & $\overline{\mathrm{H}_{3}}$ & $\mathrm{H}_{1}$ & $\mathrm{H}_{0}$ & $\overline{\mathrm{H}_{2}}$ & $\mathrm{H}_{1}$ & $\overline{\mathrm{H}_{3}}$ \\
\hline
\end{tabular}

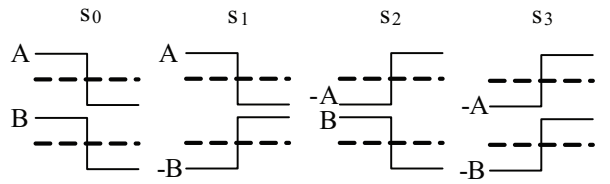

(a) Waveform constellation of $x_{0}$.

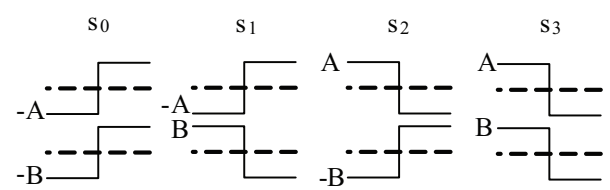

(b) Waveform constellation of $x_{1}$.

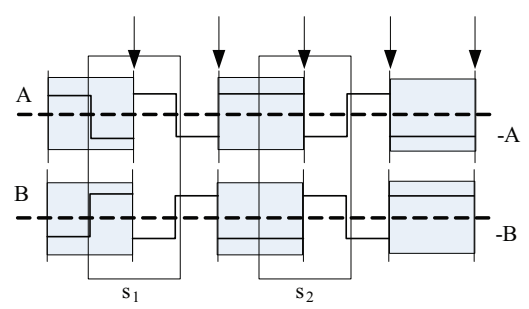

(c)

Fig. 5. Transmitted symbols for the first (left) and second (right) pairs of measurements in memory-assisted detection (upper figures). An example of two FM0 tags collision, with bit boundaries marked with arrows and two observed $\hat{x}_{1}$ half-bits marked with rectangles (lower figure).

$$
\hat{x}_{1}= \begin{cases}s_{0}, & y_{0}-y_{1}<-2 a \\ s_{1}, & -2 a<y_{0}-y_{1}<0 \\ s_{2}, & 0<y_{0}-y_{1}<2 a \\ s_{3}, & y_{0}-y_{1}>2 a .\end{cases}
$$

Erroneous detection of tag A or tag B FM0 signals occurs when detection from $\left(y_{0}, y_{1}\right)^{0}$ or detection from $\left(y_{0}, y_{1}\right)^{1}$ fails. The conditional error probabilities of such a detection scheme can be readily calculated. For example, the conditional error probability, given that $x_{0}=s_{0}$, equals:

$$
\begin{aligned}
\operatorname{Pr}\left(\hat{x}_{0} \neq\right. & \left.x_{0} \mid x_{0}=s_{0}\right)= \\
& =\int_{-\infty}^{\infty} \int_{y_{0}-2 a}^{\infty} f\left(y_{0}, y_{1} \mid x_{0}=s_{0}\right) d y_{1} d y_{0} \\
& =\int_{-\infty}^{\infty} \int_{y_{0}-2 a}^{\infty} f\left(\left[\begin{array}{r}
a+b+n_{0} \\
-a-b+n_{1}
\end{array}\right]\right) d y_{1} d y_{0} .
\end{aligned}
$$

The other three conditional error probabilities $\operatorname{Pr}\left(\hat{x}_{0} \neq x_{0} \mid x_{0}=s_{1}\right), \operatorname{Pr}\left(\hat{x}_{0} \neq x_{0} \mid x_{0}=s_{2}\right)$, $\operatorname{Pr}\left(\hat{x}_{0} \neq x_{0} \mid x_{0}=s_{3}\right)$ can be expressed similarly.

The above method requires numerical integration of the $\mathrm{Q}$ function. However, carefully observing that the method above improves the signal energy by exactly a factor of 2 , since duration of two-bits is exploited, as opposed to the aforementioned memoryless (single-bit) method, it is inferred that the error performance of the single-bit-memory assisted method improves over its memoryless counterpart with a SNR factor of two. Therefore, the probability $\operatorname{Pr}\left(\left(\operatorname{tag}_{\mathrm{A}}, \operatorname{tag}_{\mathrm{B}}\right) \neq\left(\operatorname{tag}_{\mathrm{A}}, \operatorname{tag}_{\mathrm{B}}\right)\right)$ that at least one of the two tag information is erroneously detected with the single-bit-memory assisted method is given by:

$$
\begin{aligned}
& \operatorname{Pr}\left(\left(\operatorname{tag}_{\mathrm{A}}, \operatorname{tag}_{\mathrm{B}}\right) \neq\left(\operatorname{tag}_{\mathrm{A}}, \operatorname{tag}_{\mathrm{B}}\right)\right)= \\
& =Q\left(\sqrt{2} \frac{b}{\sigma}\right)\left[2-Q\left(\sqrt{2} \frac{b}{\sigma}\right)-Q\left(\sqrt{2} \frac{a-b}{\sigma}\right)\right] \\
& +Q\left(\sqrt{2} \frac{a-b}{\sigma}\right)\left[1-\frac{1}{4} Q\left(\sqrt{2} \frac{a-b}{\sigma}\right)\right] .
\end{aligned}
$$

Simulation results confirm the calculated expression above.

Furthermore, if detection of tag A information is important while tag B detected bits can be ignored, then performance of the above method can also be calculated. Following the same reasoning as above, BER performance $\operatorname{Pr}\left(\widehat{\operatorname{tag}}_{\mathrm{A}} \neq \operatorname{tag}_{\mathrm{A}}\right)$ of the memory-assisted method, when only tag $\mathrm{A}$ is of interest, is given by Eq. (11) with SNR improved by a factor of 2 :

$$
\begin{aligned}
& \operatorname{Pr}\left(\widehat{\operatorname{tag}}_{\mathrm{A}} \neq \operatorname{tag}_{\mathrm{A}}\right)=\left[Q\left(\sqrt{2} \frac{a+b}{\sigma}\right)+Q\left(\sqrt{2} \frac{a-b}{\sigma}\right)\right] \\
& \quad \times\left\{1-\frac{1}{4}\left[Q\left(\sqrt{2} \frac{a+b}{\sigma}\right)+Q\left(\sqrt{2} \frac{a-b}{\sigma}\right)\right]\right\} .
\end{aligned}
$$

Numerical results confirm that the above expression coincides with simulation results. It is remarked that the above method does not require knowledge of the noise variance $\sigma^{2}$.

The previous two methods targeted detection at both tags, even though performance was also calculated when only tag A was of interest. In the the extended version of this work [18], ML detectors are derived when only tag A information is of interest (in the presence of tag B), with or without single-bit memory. Those detectors are omitted due to space constraints. 


\section{INVENTORY TIME BENEFITS}

In this section, the impact of the above algorithms on the reduction of total inventory time (i.e. delay) for $N$ tags is addressed. High SNR analysis follows, assuming that when exactly one or two tags transmit in a given slot their information can be correctly received.

A basic version of framed Aloha medium access scheme is assumed, since commercial RFID standards (e.g. Gen2) are based on such schemes. In the basic version of framed Aloha, access is operated in frames where each frame is divided in $L$ slots and tags at the beginning of each frame select independently and randomly one of the $L$ slots to transmit their information. At the end of the frame, the central controller (e.g. reader in the context of RFID applications) reestimates the number of remaining tags and advertises a new number $L$ of total slots for the next frame. The remaining tags select independently and randomly the slot they are going to transmit in the next frame and the process continues until a predetermined number of tags is accessed. It is remarked that for the particular case of Gen2, $L=2^{Q}$ and reader advertises $Q$ at the beginning of each frame.

For a given number $N$ of tag population and a number $L$ of slots at a given frame, the probability of $q$ tags transmitting at a given slot is described by the binomial term:

$$
\operatorname{Pr}(q)_{N, L}=\left(\begin{array}{c}
N \\
q
\end{array}\right)\left(\frac{1}{L}\right)^{q}\left(1-\frac{1}{L}\right)^{N-q} .
$$

Thus, successful transmission of tag information at a given slot can be readily calculated, also offering a measure of throughput.

First, it is assumed that tag collision occurs when more than one tags select the same slot, i.e. conventional processing at the reader. In that case, successful tag transmission occurs iff exactly one tag transmits at a slot. Throughput per slot in that case is given by:

$$
\rho_{N, L_{1}} \triangleq \operatorname{Pr}(\text { slot success })=\operatorname{Pr}(q=1)_{N, L_{1}} .
$$

Maximizing throughput per slot for a given number of slots $L_{1}$ per frame offers the appropriate number of slots which, for the case of conventional reader processing, is equal to the number of tags:

$$
\max \left\{\operatorname{Pr}(q=1)_{N, L}\right\} \Rightarrow L_{1}^{*}(N)=N .
$$

For nonconventional reader processing, e.g. when one out of two tags can be decoded at the event of simultaneous transmission of two tags (as described in Section III), throughput per slot at a frame of $L_{2}$ slots is given by

$\rho_{N, L_{2}} \triangleq \operatorname{Pr}($ slot success $)=\operatorname{Pr}(q=1)_{N, L_{2}}+\operatorname{Pr}(q=2)_{N, L_{2}}$.

Notice that, if we assumed that both tags (and not just one out of two) could be decoded at the case of simultaneous transmission of exactly two tags, then a factor of 2 would multiply the second probability term above. Maximization of the above throughput quantity offers the choice for number of slots $L_{2}$ per frame:

$$
\begin{gathered}
\max \left\{\operatorname{Pr}(q=1)_{N, L}+\operatorname{Pr}(q=2)_{N, L}\right\} \\
\Rightarrow L_{2}^{*}(N)=1+\sqrt{1+\frac{N(N-3)}{2}} .
\end{gathered}
$$

Notice that, for $N<3$ (i.e. $N=1$ or $N=2$ ), the number of slots $L_{2}^{*}=1$.

The basic framed Aloha control algorithm works as follows: maximize slot throughput per frame, i.e. set $L=L^{*}$, depending on how tag collision is defined (whether aforementioned detection algorithms of Section III are applied, in which case $L=L_{2}^{*}(N)$ or not, in which case $\left.L=L_{1}^{*}(N)\right)$. When frame is completed (i.e. all slots are tested), update number $N$ of backlogged tags (remaining number of tags to be read) and start a new frame.

It is remarked that the above algorithm assumes that the central controller (e.g. reader) has acquired an accurate estimate of the total number of tags $N$. Such information can be inferred from the number of empty or collided slots and there are specific proposals in the literature, based on deterministic [7], probabilistic [19], [20], or recursive [21] techniques. More importantly, the above policy maximizes throughput per frame and not total number of frames (overall delay). It was recently shown that it could be beneficial to stop a frame before the total number of slots is tested (especially when probability of tag transmitting at remaining slots is small) and start a new frame with an updated slot number [22], [23]. Optimizing the framed Aloha policies are beyond the scope of this work.

The expected total number of frames $F$ and expected total number of slots, required for the aforementioned basic framed Aloha scheme, can be readily calculated with the recursive equations (33)-(35) below, with initial condition $N(1)=N$.

$$
\begin{gathered}
L(i)=L^{*}(N(i)), \\
N(i+1)=N(i)-L(i) \rho_{N(i), L(i),} \\
\sum_{i=1}^{F} L(i) \rho_{N(i), L(i)} \geq a N .
\end{gathered}
$$

The first equation sets the number of slots per frame according to Eq. (30) or Eq. (32), depending on the reader detection method. The second equation computes the expected number of remaining tags at the end of the frame, which is used to calculate the number of slots for the next frame. The third expression sums all accessed tags and terminates the recursion if their sum is above the percentage $a$ of the total tags that need to be read.

With the above recursion, the expected total number of frames $F$ and slots per frame $L(i)$ are estimated, when Eq. (30) or Eq. (32) are utilized. In both cases, the expected total number of slots required to access $a N$ tags (e.g. $a=$ $100 \%=1$ ) is given by:

$$
\sum_{i=1}^{F} L(i) .
$$


Simulation results of the basic framed Aloha scheme confirm the theoretical calculation above, as will be shown at Section V. With the above recursive methodology, inventory time benefits (i.e. delay reduction) can be readily calculated when detection techniques for two collided tags are utilized, as opposed to conventional detection (where collided signals of two tags are discarded).

\section{A. Implication to Gen 2}

Particularly in Gen2, the population of tags to be accessed is first selected. Then, the protocol enters the query phase, where tags contend for the channel using framed Aloha and random IDs of 16-bit length. After successful acknowledgment of a single tag's 16-bit ID by the reader (i.e. singulation), the singulated tag transmits its 96-bit electronic product code (EPC) in the access phase of the protocol. In that phase, the reader can also access other portions of the tag memory. Therefore, tag contention is restricted during the query phase where tag-transmitted information has different duration (i.e. smaller) than the information transmitted during access where only a single tag transmits.

Thus, the overall time required to access a population of $N$ tags in Gen2 can be calculated as:

$$
\begin{aligned}
& \mathcal{I}_{\text {Gen2 }}=\left(\sum_{i=1}^{F} L(i)\right) \mathcal{T}_{\text {singulation slot }}+N \mathcal{T}_{\text {EPC slot }} \\
& =\left(N+\beta \sum_{i=1}^{F} L(i)\right) \mathcal{T}_{\text {EPC slot }}, \\
& \beta=\mathcal{T}_{\text {singulation slot }} / \mathcal{T}_{\text {EPC slot }},
\end{aligned}
$$

where $\mathcal{T}_{\text {singulation slot }}$ is the expected slot duration of the framed Aloha protocol during tag contention (query phase), $\mathcal{T}_{\mathrm{EPC} \text { slot }}$ is the overall time needed to transmit the EPC code during the access phase (when only the singulated tag transmits), and $\beta$ is their ratio. Both slot durations include necessary preambles, cyclic redundancy check (CRC), end bits, as well as duration of flag and acknowledgment packets transmitted by the reader. Such preambles or packet durations depend on the Gen 2 operating point, controlled by the reader.

A crude approximation for $\beta$ (that ignores preambles, CRC protection, and reader acknowledgments) can be found if one considers that tag ID has duration of 16 bits during query and 96 bits during EPC access; thus, $\beta \simeq 16 / 96=16 \%$. In practice, and depending on the selected transmission rates from tag to reader and reader to tag (which are in general variable, reader-controlled, and different), $\beta$ can reach the values of $\beta=22 \%$ or $\beta=27 \%$. Gen2 details are beyond the scope of the this work. However, it is remarked that the above value of $22 \%$ is attained for Tari $=6.25 \mu \mathrm{sec}$, $\mathrm{RTcal}=2.5 \mu \mathrm{sec}$, TRcal $=33.3 \mu \mathrm{sec}, \mathrm{DR}=8$, including preambles, $\mathrm{CRC}$ protection, acknowledgments, and end bits. For $\mathrm{DR}=64 / 3$ and all the rest kept the same, $\beta=27 \%$. In the numerical results section, $\beta$ is left as an input parameter in $[0.1,0.3]$.

According to the above, the overall inventory time reduction (in percentage), when a Gen2 reader is equipped with the

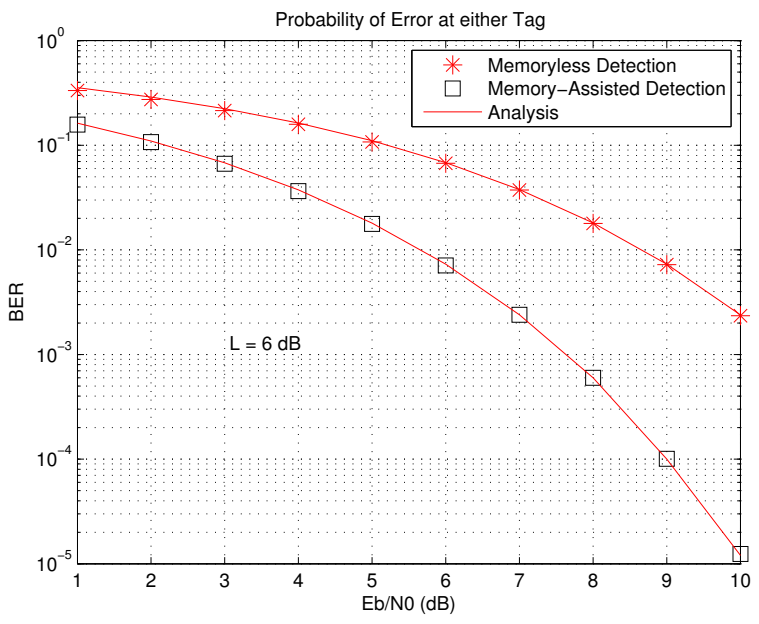

Fig. 6. BER at either tag vs SNR (fixed $L=6 \mathrm{~dB}$ ).

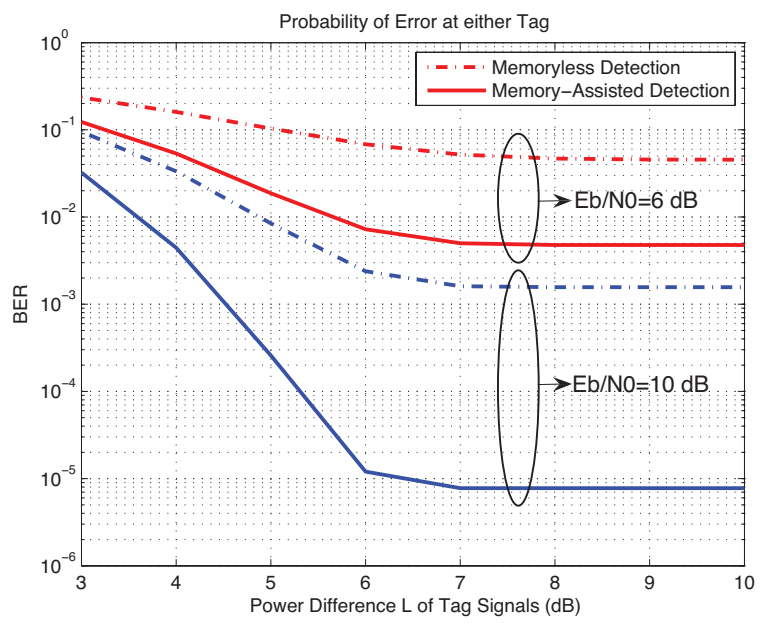

Fig. 7. BER at either tag vs tag power difference L (fixed SNR).

detection methods above, allowing successful identification of one out of two collided tags, is given by

$$
\begin{aligned}
& \frac{\beta\left[\sum_{i} L_{1}(i)-\sum_{j} L_{2}(j)\right]}{N+\beta \sum_{i} L_{1}(i)} . \\
& \text { V. NUMERICAL RESULTS }
\end{aligned}
$$

In our simulation results, we make use of the signal-tonoise ratio (SNR) $E_{b} / N_{0}=b^{2} / \sigma^{2}$ as well as the power ratio between the two baseband tag signals $L=a^{2} / b^{2}$.

In Fig. 6, the BER as a function of SNR is depicted, when detection error at either tag (A or $\mathrm{B}$ ) is considered. The power difference between the two tags is set to $L=6 \mathrm{~dB}$ (i.e. $a=$ $2 b$ ) and the two detection methods (memoryless vs memoryassisted of subsections III-A-III-B) are tested.

It is found that simulation matches analytical results. Furthermore, the memory-assisted method performs $3 \mathrm{~dB}$ better than its memoryless counterpart, due to intelligent exploitation of FM0 memory, as explained in Subsection III-B. 


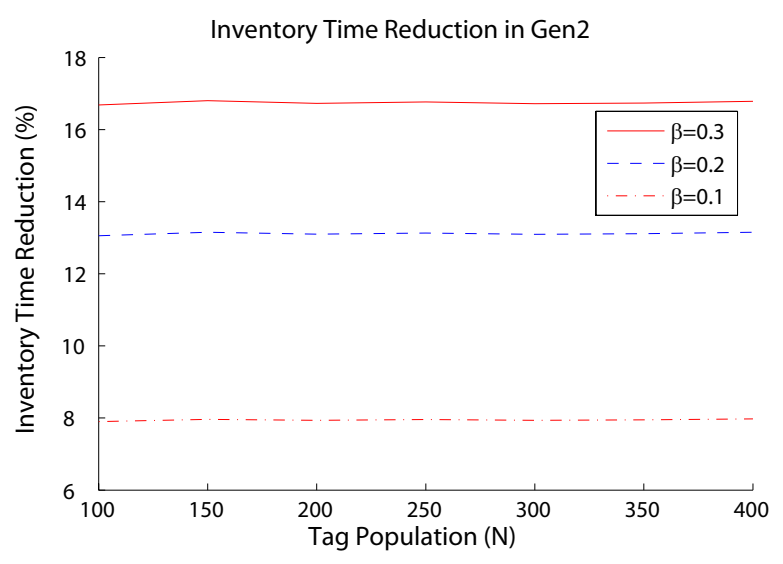

Fig. 8. Reduction of inventory time in Gen2 (Eq. (39)).

In Fig. 7, the previous experiments are repeated with fixed SNR and variable $L$. As $L$ increases, the overall BER reaches a plateau. That is due to the fact that error at either tag is considered and, thus, the depicted BER is limited by the weakest tag (B in our case); by increasing $L$, errors at the strongest tag (tag A) are decreased but errors at the weakest tag are left unaffected. Thus, in cases where there is collision with a "weak" tag, the reader should only focus on the stronger tag. Such strategy has been examined in [18] (results are omitted due to space constraints). It was found that the memoryassisted detection method could drop BER to values on the order of $10^{-6}$ for SNR close to $10 \mathrm{~dB}$ and $L=6 \mathrm{~dB}$. One immediate question emerges: could additional FM0 memory (more than one bit) further reduce BER? The answer is negative and was already given by Simon and Divsalar for single-tag detection [14].

In Fig. 8, the reduction of inventory time in Gen2 is calculated through Eq. (39) for three different values of $\beta$. It can be seen that total inventory time in Gen2, using single antenna techniques (as in this work), can be reduced by approximately $8 \%, 13 \%$, and $17 \%$ for $\beta=0.1,0.2$, and 0.3 , respectively. Such Gen2 percentage is reduced compared to what was observed at the basic framed Aloha protocol, as already explained in Section IV. In any case, the calculated reduction is still substantial. Similar reduction figures of Gen2 total inventory time on the order of $10 \%$, when extraction of a single tag information out of a slot with two collided tags, have been also reported in [13] with a different calculation approach.

\section{CONCLUSION}

The detection techniques of this work, destined for collided FM0 tags can benefit framed Aloha-based RFID systems, including Gen2. Memory-assisted detection of collided FM0 signals for a tag population $N$ could lead to inventory time reduction on the order of $8-17 \%$ for existing Gen 2 access schemes. It is remarked that no modification of the existing readers' RF front end is required, as the proposed techniques could operate with single-antenna readers. This might be important for portable UHF Gen2 RFID systems, where multiple- antennas are not always an option. The techniques can be also applied on multi-antenna readers operating in antenna switching mode.

\section{REFERENCES}

[1] H. Stockman, "Communication by means of reflected power," Proc. IRE, pp. 1196-1204, 1948.

[2] K. Finkenzeller, RFID Handbook: Fundamentals and Applications in Contactless Smart Cards and Identification, 2nd ed. New York: John Wiley and Sons, 2003.

[3] G. Vannucci, A. Bletsas, and D. Leigh, "A software-defined radio system for backscatter sensor networks," IEEE Trans. Wireless Commun., vol. 7, no. 6, pp. 2170-2179, June 2008.

[4] A. Bletsas, S. Siachalou, and J. N. Sahalos, "Anti-collision backscatter sensor networks," IEEE Trans. Wireless Commun., vol. 8, no. 10, pp. 5018-5029, Oct. 2009.

[5] C. M. Kruesi, R. J. Vyas, and M. M. Tentzeris, "Design and development of a novel 3D cubic antenna for wireless sensor networks (WSN) and RFID applications," IEEE Trans. Antennas Propagat., vol. 57, no. 10, pp. 3293-3299, Oct. 2009.

[6] EPC Radio-Frequency Identity Protocols, Class-1 Generation-2 UHF RFID Protocol for Communications at $860 \mathrm{MHZ}-960 \mathrm{MHZ}$, version 1.2.0. EPC Global, 2008.

[7] F. C. Schoute, "Dynamic frame length aloha," IEEE Trans. Commun., vol. 31, no. 4, pp. 565-568, Apr. 1983.

[8] J. E. Wieselthier, A. Ephremides, and L. A. Michaels, "An exact analysis and performance evaluation of framed ALOHA with capture," IEEE Trans. Commun., vol. 31, no. 2, pp. 125-137, Feb. 1989.

[9] G. R. Woo, "Demonstration and evaluation of co-channel DBPSK source separation," M.S. Thesis, MIT, 2007, mentor Andrew Lippman.

[10] D. Shen, G. Woo, D. P. Reed, A. B. Lippman, and J. Wang, "Separation of multiple passive RFID signals using software defined radio," in Proc. IEEE RFID Conf., Orlando, FL, Apr. 2009.

[11] C. Angerer, R. Langwieser, and M. Rupp, "Rfid reader receivers for physical layer collision recovery," IEEE Trans. Commun., vol. 58, no. 12, pp. 3526-3537, Dec. 2010 .

[12] A. F. Mindikoglu and A.-J. van der Veen, "Separation of overlapping RFID signals by antenna arrays," in ICASSP, 2008, pp. 2737-2740.

[13] B. Frey, "Source separation for uhf rfid," M.S Thesis, Delft and ETH, 2008, mentor Geert Leus, supervisor Helmut Bölcskei.

[14] M. Simon and D. Divsalar, "Some interesting observations for certain line codes with application to rfid," IEEE Trans. Commun., vol. 54, no. 4 , pp. 583-586, Apr. 2006

[15] M. Buettner and D. Wetherall, "A "Gen 2" RFID monitor based on the USRP," ACM SIGCOMM Computer Communication Review, vol. 40, no. 3, pp. 42-47, July 2010.

[16] D. M. Dobkin, The RF in RFID: Passive UHF RFID in Practice. Newnes (Elsevier), 2008.

[17] A. Bletsas, A. G. Dimitriou, and J. N. Sahalos, "Improving backscatter radio tag efficiency," IEEE Trans. Microwave Theory Tech., vol. 58, no. 6 , pp. 1502-1509, June 2010.

[18] A. Bletsas, J. Kimionis, A. G. Dimitriou, and G. N. Karystinos, "Singleantenna coherent detection of collided FM0 RFID signals," submitted for publication, Apr. 2011, also archived as TUC Telecom Lab Technical Report.

[19] H. Vogt, "Efficient object identification with passive RFID tags," in PERVASIVE 2002. Zurich, Switzerland: F. Mattern and M. Naghshineh, editors, volume 2414 of Lecture Notes in Computer Science (LNCS), Aug. 2002.

[20] C. Floerkemeier, "Bayesian transmission strategy for framed ALOHA based RFID protocols," in Proc. IEEE RFID Conf., Grapevine, TX, Mar. 2007.

[21] J.-B. Eom and T.-J. Lee, "Accurate tag estimation for dynamic framedslotted ALOHA in RFID systems," IEEE Commun. Lett., vol. 14, no. 1, pp. 60-62, Jan. 2010.

[22] L. Zhu and T.-S. P. Yum, "Design and analysis of framed aloha based RFID anti-collision algorithms," in Proc. IEEE Global Telecommunications Conference (Globecom), Honolulu, HI, Nov. 2009.

[23] — , "Optimal framed aloha based anti-collision algorithms for RFID systems," IEEE Trans. Commun., vol. 58, no. 12, pp. 3583-3592, Dec. 2010. 